\title{
Las dinámicas de expansión del capital y el capitalismo cognitivo: algunos de sus posibles influencias en la educación superior
}

\author{
Nayihe Peña Frade* \\ Recibido: 4 de mayo de 2017 \\ Enviado a pares evaluadores: 19 de mayo de 2017 \\ Aprobado por pares evaluadores: 23 de junio de 2017 \\ Aprobado por Comité Editorial: 6 de julio de 2017 \\ DOI: 10.22395/csye.v6n12a5
}

\section{RESUMEN}

En este artículo se plantea al capitalismo como el marco económico y productivo global que está en el origen, la base y el horizonte de algunos enunciados que pesan sobre las actividades universitarias, entre ellos, que la educación superior es estratégica en la competitividad de las naciones, que las universidades deben producir conocimiento útil con respecto a las múltiples necesidades de la sociedad y que, para cumplir esos propósitos, las instituciones y programas deben ser de alta calidad. Para acometer esta temática compleja, se analizan tres tópicos: la dinámica de expansión de la ganancia, el uso intensivo de tecnología y el capitalismo cognitivo, resultado de los anteriores. El artículo es producto de la investigación doctoral " $\mathrm{El}$ Sistema de Aseguramiento de la Calidad de la Educación Superior (Saces) y las políticas públicas para la educación superior en Colombia (1970-2010): continuidades y rupturas", que realiza la autora.

Palabras claves: capitalismo; gestión del conocimiento; política gubernamental; propiedad intelectual; Estado y educación; enseñanza superior.

Doctoranda Universidad Arcis, doctorado Cultura y Educación en América Latina. Magíster Universidad Nacional de Colombia, maestría en Urbanismo. Socióloga Universidad Nacional de Colombia. Docente medio tiempo del programa de Historia, Universidad Autónoma de Colombia. Hace parte del grupo de investigación Grafía: Sociedad, Pensamiento e Imaginación, línea Humanidades y Educación de la Universidad Autónoma de Colombia. Correo electrónico: nayibe.pena@fuac.edu.co 


\section{Dynamics of capital expansion and cognitive capitalism: some possible influences on higher education}

\section{ABSTRACT}

In this article, capitalism is posed as the economic and productive global framework that is at the origin, base and horizon of some statements influencing university activities, among them, that higher education is strategic in nations competitiveness, that universities should produce useful knowledge regarding the multiple needs of society and that, in order to fulfill these purposes, institutions and programs must be of high quality. In order to tackle this complex subject, three topics are analyzed: the dynamics of profit expansion, the intensive use of technology and cognitive capitalism. The paper is the product of the author's PhD. research "Higher Education Quality Assurance System -HEQAS- and Public Policies for Higher Education in Colombia 1970-2010; Continuities and Ruptures" (El Sistema de Aseguramiento de la Calidad de la Educación Superior -Saces- y las políticas públicas para la educación superior en Colombia (1970-2010): continuidades y rupturas).

Keywords: capitalism; knowledge management; government policy; intellectual property; state and education; higher education.

\section{As dinâmicas de expansão do capital e o capitalismo cognitivo: algumas de suas possiveis influências no ensino superior}

\section{RESUMO}

Neste artigo, apresenta-se o capitalismo como o referencial econômico e produtivo global que está na origem, base e horizonte de alguns enunciados que tratam das atividades universitárias, entre eles, que o ensino superior é estratégico na competitividade das nações, que as universidades devem produzir conhecimento útil a respeito das múltiplas necessidades da sociedade e que, para cumprir com esses propósitos, as instituições e os programas devem ser de alta qualidade. Para empreender essa temática complexa, analisam-se três tópicos: a dinâmica de expansão do lucro, o uso intensivo de tecnologia e o capitalismo cognitivo, resultado dos anteriores. $\mathrm{O}$ artigo é produto da pesquisa de doutoramento "O sistema de garantia da qualidade do ensino superior (Saces) e as políticas públicas para o ensino superior na Colômbia (1970-2010): continuidades e rupturas", que a autora realiza.

Palavras-chave: capitalismo; educação superior; Estado e educação; gestão do conhecimento; política governamental; propriedade intelectual. 


\section{Introducción}

A partir de los años noventa, y en sintonía con las transformaciones productivas, tecnológicas, institucionales y políticas de distinto calado que se estaban produciendo al nivel global, los gobiernos nacionales se empeñaron en promover y consolidar la innovación, la competitividad y la productividad de la economía colombiana en el mundo y aumentar las exportaciones con alto valor agregado. Consideraban los gobernantes que la competitividad y la productividad dependían de que aumentaran la escolaridad, la pertinencia y la capacidad del capital humano para innovar, y en pos de ambos cometidos, el de competitividad y de formación de la fuerza de trabajo más calificada, las políticas para la educación superior se centraron en la definición e implementación de estándares de cali$\operatorname{dad}^{1}$. Se esperaba también que una educación superior de calidad propiciara el emprendimiento empresarial, promoviera la apropiación social del conocimiento y aumentara el uso de tecnología. Para lograr estos resultados las universidades debían procurar la congruencia entre las necesidades de capital humano del sector productivo y los perfiles ocupacionales de los egresados. Se generalizó la idea de que la contribución de la educación superior al desarrollo estaba dada por la efectividad con que este aprendizaje fuera aplicable, fuera "conocimiento útil"2.

En el origen de los cambios significativos de la institución universitaria y la educación superior hubo cinco presiones externas: (i) la urgencia por tener una economía más competitiva en el mercado internacional; (ii) la importancia adquirida por la oferta internacional de educación superior que ponía en desventaja a la oferta pública y privada nacional; (iii) la creciente influencia de organismos inter y supranacionales con discursos modernizadores y globalizantes; (iv) el aumento de las prescripciones, regulaciones y formas de control del Estado sobre las instituciones universitarias; y (v) la presión conjugada de las anteriores en torno a la consecución de la calidad. Con el paso de los años estas presiones no disminuyeron, sino que, por el contrario, hicieron evidente el papel crítico que tienen las universidades en los esfuerzos del Estado "por competir en la economía mundial del conocimiento, así como en satisfacer la necesidad del sector productivo de contar con una fuerza de trabajo globalizada" (Ordorika y Lloyd, 2014, p. 125).

Sobre los nudos problemáticos del concepto y los estándares de calidad puede consultarse Peña Frade (2015).

2 Esta definición del problema universitario tiene una larga trayectoria y hay una copiosa bibliografía al respecto $\mathrm{El}$ documento del Banco Mundial (2003) incluye varios estudios que han sido ampliamente referenciados en dicha bibliografía. El documento de la Asociación Colombiana de Universidades, Ascun (2007), brinda una apretada visión general de los problemas más importantes de las instituciones universitarias nacionales. También es importante el análisis de Unesco-Cresalc, Ascun (1998). 
Si bien la explicación anterior es válida, no es suficiente porque está arraigada en un contexto histórico, político y social local, acotado e inmediato, y deja en la sombra a un sistema más amplio y complejo: las dinámicas del capitalismo global que han incidido en las mutaciones que está teniendo el sistema universitario en el mundo; en ese plano se encuentra a financiadores, agencias y corporaciones que se mueven en el ámbito de la producción y comercialización del conocimiento, y que muestran que la universidad hace parte de una relación entre educación y mercado, entre conocimiento, saber y poder.

Este artículo pretende explicitar algunas de esos cambios globales, más exactamente los que terminaron dando origen a políticas de producción de saber y conocimiento y que inspiran modelos universitarios. La educación superior en Colombia fue reestructurada a través de políticas públicas³; está por dilucidarse con precisión qué tanto, y cómo, incidieron esas políticas globales en la transformación interna, y está pendiente la valoración de los efectos que pueda tener la adopción de los estándares internacionales de calidad que las orientan. A continuación, se exponen tres rasgos del capitalismo global que pueden tener gran ascendiente en las políticas públicas de aseguramiento de la calidad del sistema de educación superior colombiano, y en la reforma de las universidades. Entonces, con la pretensión de proponer un marco de análisis complejo para sopesar las políticas públicas orientadas a la calidad de la educación superior se desarrollan los siguientes temas: (i) la expansión global del capital y la preponderancia del capital financiero en esa dinámica; (ii) la tecnología como capital constante, y (iii) el capitalismo cognitivo.

\section{Metodología}

La investigación doctoral "El Sistema de Aseguramiento de la Calidad de la Educación Superior (Saces) y las políticas públicas para la educación superior en Colombia (1970-2010): continuidades y rupturas" se propone hacer una revisión crítica de las políticas públicas que han promulgado doce gobiernos colombianos sobre la educación superior, en un período de cuarenta años. Son tres las hipótesis que se exploran en la investigación:

1. El Saces representa una ruptura con las políticas para la educación superior previas a su proclamación, y dominantes en las décadas del setenta y el ochenta ${ }^{4}$.

3 Un documento pertinente sobre dichas políticas en América Latina es Los temas críticos de la educación superior en América Latina en los años noventa. FCE, Universidad Autónoma de Aguascalientes y FLACSO, Chile. México, 2002.

4 Esta hipótesis tiene su origen en un análisis de los planes de desarrollo de los gobiernos del período 19581978 que mostró que las nociones que tenían esos gobiernos acerca de la función y la importancia de las universidades era, en varios sentidos, diferente a las que fundamentan las políticas más recientes. Consultar Peña Frade (2013). 
2. La mayoría de las razones que explican el cambio de la orientación en las políticas públicas en Colombia se inscribe dentro de la consolidación definitiva de una economía globalizada, así como en el propósito de superar rezagos y carencias de la producción de bienes y servicios, y la inadecuación de la fuerza de trabajo existente en el país para superar esa situación de atraso.

3. El capitalismo cognitivo, basado en el uso del conocimiento como factor fundamental en la producción, el consumo y la competencia es la racionalidad que impera en la educación superior, y es el criterio que ha orientado la trasformación de las universidades y que hizo posible que se concretara el SACES.

Durante el proceso se ha venido construyendo una argumentación que permita responder las preguntas que orientan la investigación: ¿Desde qué argumentos se pueden identificar las continuidades y rupturas entre el Saces y las políticas públicas para la universidad colombiana que lo precedieron? ¿Establecer y explicar tales rupturas permite conocer las razones que motivaron el Saces y la racionalidad que anima al aseguramiento de la calidad? ¿Cómo puede caracterizarse la noción de calidad que sirve de eje articulador de las políticas educativas?

La revisión de fuentes primarias y secundarias ha generado diversas etapas de esa argumentación. En un principio la reflexión estuvo centrada en la relación entre universidad, actividad académica y actores internos; después se incorporó el deber ser de la institución con respecto al beneficio de la sociedad. Este era un enfoque funcionalista centrado en los procesos internos de gestión y de toma de decisiones (Ordorika y Lloyd, 2014, p. 127).

Más adelante el foco de la reflexión se amplió para definir y formular el problema desde la noción de políticas públicas y dentro de estas, las políticas para la educación superior en Colombia; en esta fase el análisis consideró la política pública como acción de gobierno dentro de un régimen político, determinado, todo ello, por la reestructuración de los Estados nacionales en respuesta a los desafíos de la economía y la producción globalizada. Se asumió que la forma como la globalización de la producción, la economía, el comercio, la cultura y la política, le han ido imprimiendo a los Estados nacionales, a la relación entre Estado, Sociedad y Gobierno, y a las acciones gubernamentales dentro de un régimen político determinado ha producido a una transformación de fondo en la educación superior: varió, de forma sustancial o parcial, la visión de las universidades acerca de su responsabilidad o su función con respecto a la sociedad nacional en la cual tuvieron su origen, y en la que han venido operando desde su creación. Este giro es resultado de varios factores, el de mayor envergadura quizá sea el primado de la economía de mercado, de la ciencia y de la tecnología, 
predominio que se apoya en una presión ideológica, comercial y militar que empuja el fortalecimiento constante de la especialización dirigida al mercado mundial, y la inserción más intensa al sistema financiero internacional (Esser et al., 1994, p. 2).

Esa fase se centró en la presión externa proveniente de un entorno hostil ante la cual las universidades habrían desplegado estrategias adaptativas orientadas a garantizar su supervivencia (Ordorika y Lloyd, 2014, p. 128); las formas que adquirieron esas estrategias mostrarían tanto las transformaciones mismas de la universidad como los impactos de dichos cambios. Las limitaciones de ese enfoque radican en que sigue centrado en el interior de la universidad abstrayéndola de su entorno político, y en que excluye la posibilidad de que las instituciones de educación superior (IES), considerando las oportunidades que podría representar ese entorno externo que las presionaba, hayan optado, y no solo reaccionado, a la hora de tomar decisiones y emprender acciones. Estas limitaciones impiden, en primer lugar, considerar la posibilidad de que haya universidades que, por alguna razón, no buscan adaptarse o dar respuesta a las presiones (Ordorika, 2001, p. 79); segundo, dilucidar el papel que cumple la tensión entre una cultura de responsabilidad o compromiso social y otra gerencial-administrativa, ambas con fuerte presencia no solo en las universidades sino en el Estado mismo.

Explicar las transformaciones estructurales de la educación superior como la reacción de las instituciones a un entorno adverso dificulta, en tercer lugar, algo muy importante: ver a la universidad dentro de un campo en el cual hay otros actores y en el que se disputa en torno a posiciones, es decir, verlas dentro de un "entramado global [...] como un campo existente a nivel mundial" (Ordorika, 2006, p. 38). Este campo universitario mundial da forma a un

[...] sistema único de educación superior mundial: una red de páginas web, unidas por mensajes electrónicos instantáneos y transferencia de datos, en la que conexiones globales corren por el centro de instituciones educativas y gobiernos y son componentes integrales de las prácticas cotidianas. Al mismo tiempo la movilidad global de personas en la educación se ha incrementado sustancialmente. Por su parte las comunicaciones globales y la movilidad han creado las condiciones para la emergencia de un mercado global en la educación superior. Este mercado global está caracterizado por la competencia entre las instituciones más prestigiadas a nivel mundial y es cada vez más cercano a las formas económicas capitalistas (Ordorika, 2006, p. 39).

Ese mercado, como dice Ordorika, se configura como un sistema de poder (Ordorika, 2006, p. 39).

De esa forma se amplía el campo de la investigación y se empieza a considerar que "las organizaciones de educación superior son espacios políticos en los que 
se confrontan visiones y proyectos alternativos" (Ordorika, 2001, p. 81). Además, permite conducir la argumentación a un punto en el cual se puedan proponer ideas relevantes acerca de tensiones de fondo como la necesidad de competir en un medio universitario globalizado y la responsabilidad social que tiene la institución universitaria en su respectivo medio nacional, la autonomía de instituciones y profesionales y la regulación del Estado o la presión del mercado (Ordarika, 2006, p. 36).

Fue en ese marco donde se destacó la necesidad de sopesar tres asuntos medulares en la globalización y de dilucidar su importancia y su papel en relación con el campo mundial universitario. Esos tópicos son, como se indicó atrás, las dinámicas de expansión global del capital y la preponderancia del capital financiero, el uso que se da a la tecnología como factor de producción en la forma de capital constante y el capitalismo cognitivo como la circulación de conocimientos dentro de los procesos de producción, creación de valor y consumo.

\section{Resultados}

\section{Dinámica expansionista del capitalismo actual}

La noción de capitalismo implica la

[...] exigencia de acumulación ilimitada de capital a través de medios formalmente pacíficos. La perpetua puesta en circulación del capital dentro del circuito económico con el objetivo de extraer beneficios, es decir, de incrementar el capital que será a su vez reinvertido de nuevo, sería lo que caracterizaría al capitalismo y lo que le conferiría [su] dinámica y fuerza de transformación (Boltanski y Chiapello 2002, p. 3).

El propósito del sistema económico conocido como capital es la acumulación de riqueza, y su necesaria consecuencia es su auto-expansión (Mignolo s. f., p. 21). El incremento que está en la esencia del capitalismo se traduce en la exigencia de aumentar permanentemente la tasa de ganancia de los capitalistas y las empresas (Amin 1999, p. 30).

Dicho agrandamiento supone, primero, que exista una demanda solvente y diferenciada, que esté en capacidad social, intelectual y actitudinal de ser satisfecha por empresas, establecimientos e instituciones; segundo, una implantación ventajosa de los modos de producción, distribución y consumo en los países y mercados de centro y periferia, lo cual incluye, tercero, el abaratamiento de la fuerza de trabajo.

Las sociedades contemporáneas y su modo de vida urbano, globalizado, hipercomunicado por redes y tecnologías de comunicación, productor y consumidor de toda suerte de información, generan muchas oportunidades para que 
se amplíen y profundicen las relaciones de intercambio, mercantilización que sucede ahora con la conversión de actividades sociales e individuales en negocios, a través de los cuales el capital produce la oferta que requiere la demanda. En este proceso entran la subjetividad y la sensibilidad de las personas porque "su "necesidad de conocer" y el "amor a lo bello y la avidez de lo exquisito" son los grandes estuarios que se abren al desarrollo del progreso económico" (Lazzarato 2004, p. 130). Nada queda por fuera de esa creciente mercantilización de la existencia, ni siquiera la sensibilidad puesto que "A medida que las necesidades se tornan cada vez más específicas, el valor estético se convierte en uno de los elementos fundamentales y estimuladores del deseo de producción y del deseo de consumir" (Lazzarato 2004, p. 138).

La proliferación de las nuevas tecnologías de información y comunicación (NTIC) representa una situación promisoria para la expansión del capital por cuanto el desarrollo de tecnología desencadena constantes procesos de innovación social y en la producción, que promueven la competitividad y el desarrollo social, garantizando así los patrones de consumo convenientes para mantener el crecimiento (Martínez 2012, p. 30).

Pero lo más importante es que el aumento de la tasa de ganancia que está en la base de la expansión del capital depende de la disminución sostenida del valor del trabajo vivo y del aumento de capital constante en detrimento del capital variable, fin para el cual la tecnología es un medio. Como plantea Marx:

\footnotetext{
En la medida en que la gran industria se desarrolla, la creación de la riqueza efectiva se vuelve menos dependiente del tiempo de trabajo y del cuanto de trabajo empleados, que del poder de los agentes puestos en movimiento durante el tiempo de trabajo, poder que a su vez no guarda relación alguna con el tiempo de trabajo inmediato que cuesta su producción, sino que depende más bien del estado general de la ciencia y del progreso de la tecnología, o de la aplicación de esta ciencia a la producción (Marx 2006, p. 7).
}

Las condiciones, ya no para simplemente depreciar la fuerza laboral, sino para reemplazar trabajo vivo se han hecho óptimas en el capitalismo contemporáneo, momento en el cual "el mecanismo de acumulación fundado en la producción de mercancías materiales ha cambiado a favor de la posibilidad de multiplicar sin límites mercancías inmateriales prácticamente sin costos agregados" (Calderón 2008, p. 156).

Por otro lado, en la economía globalizada se ha venido produciendo una progresiva sustitución del capital nacional por el capital mundializado, así como del capital productivo por el financiero como sector dominante (Dussel 2014, p. 312). Los actores fundamentales en el sistema financiero global del orden neoliberal son: (i) los centros hegemónicos, básicamente los Estados Unidos y la Unión Europea; (ii) los organismos intergubernamentales de negociación y 
de crédito que administran y difunden estas reglas de juego, y (iii) las corporaciones trasnacionales, que son las principales beneficiarias del nuevo tipo de transacciones globales que han proliferado tras la instalación de las tecnologías de la información y de las comunicaciones (Di Filippo 2009, p. 200).

Este relevo conlleva una dependencia aún mayor de las economías nacionales de los capitales internacionales, con el consecuente aumento de su vulnerabilidad a las crisis financieras globales; una pérdida de influencia de los Estados y las empresas en la conformación del panorama financiero internacional; sociedades, Estados y economías carentes de medios e instrumentos de carácter preventivo o por lo menos protector; menoscabo de la soberanía en la toma de decisiones políticas referentes al tipo de cambio, inversión de divisas, comercio internacional y decisiones crediticias en los sectores público y privado, entre otras.

El panorama resultante puede ser sintetizado como uno en el cual las crisis económicas son gestionadas por el capitalismo en un espacio cada vez más mundializado, y las crisis sociales y políticas en espacios nacionales diferentes (Amin, 1999, p. 49). La sustitución de la propiedad nacional le da una dimensión mundial y nueva a la competencia entre capitalistas y trabajadores (Martínez, 2001, p. 27). La actividad económica que se realiza en y para un marco supranacional implica decisiones que no toman agentes definidos por su carácter nacional; está regida por mecanismos inaccesibles e inmanejables para las instituciones definidas a escala nacional, local o regional (Martínez, 2001, p. 9).

\section{Tecnología, trabajo, producción y trabajadores}

La tecnología es un proceso y una relación que implica y envuelve al tecnólogo, a la ciencia y al usuario del producto (Maldonado, 2001, p. 27); es la expresión cotidiana de la ciencia (Maldonado, 2001, p. 28). La globalización es el resultado y el proceso de la era tecnológica.

Enrique Dussel define tres momentos dialécticos de la tecnología: el primero es el de tecnología en sí donde se sitúan el tecnólogo o el ingeniero; es abstracto porque hace caso omiso de determinaciones reales que, como la ideología, la política y la economía, pesan sobre ella. En el segundo momento la tecnología es considerada como un instrumento de trabajo, como parte de un todo, como una mediación en y para la producción ${ }^{5}$. Y, por último, la tecnología es un momento esencial y fundamental del capital en general (Dussel, 2014, p. 356). La tecnología es capital constante porque está en su origen, en el permanente mejoramiento

\footnotetext{
5 En este segundo momento de la tecnología se localizarían el análisis de las relaciones entre universidad, sector productivo y gobierno basado en el caso de México que hace Casalet (2006), y el modelo de la triple hélice de Etzkowiz (2009) que se mencionan más adelante.
} 
de los instrumentos de trabajo para que produzcan un aumento cuantitativo de la productividad (Dussel 2014, p. 359) y, con ella, del lucro. Puesto que el conocimiento es un bien inmaterial que aumenta su valor a través del uso y la difusión, al contrario de los bienes materiales que se desgastan o deterioran (Guzmán 2008, 48), la tecnología, en la cual hay conocimiento incorporado y trabajo objetivado es, en sí misma, una fuente de valor; por tanto, aumenta la ganancia de las empresas que la incorporan en su producción porque pueden vender su mercancía a un precio final mayor de su valor.

El aumento de la proporción del capital constante implica la disminución del peso del capital variable, que está representado en el trabajo vivo, y precipita mutaciones en la fuerza de trabajo. Exige de ella una mayor y específica calificación, la somete a una explotación más intensiva, precariza sus condiciones laborales, instala una relación de competencia y rivalidad entre los trabajadores lo cual intensifica y asegura su atomización como actor social. La producción de mercancías inmateriales

[...] ha cambiado en dos formas la naturaleza del trabajo asalariado: por un lado, la creación de una miríada de nuevos trabajos, oficios, profesiones y especializaciones que han fragmentado la figura del trabajador en un caleidoscopio de funciones y tareas $y$, por el otro, ha descentralizado la producción en una multiplicidad de lugares y espacios distintos, gracias al carácter inmaterial de la información, que no exige transporte físico y puede ser transmitida a grandes distancias". Se ha ido suprimiendo la división entre trabajo productivo e improductivo y con ella la figura social antagonista de la relación capitalista dentro y fuera de la fábrica (Calderón 2008, p. 156).

Esta sustitución de trabajo vivo, dice Marx, trae consecuencias importantes para individuos y sociedades:

El trabajo inmediato se ve reducido cuantitativamente a una proporción más exigua, y cualitativamente a un momento sin duda imprescindible, pero subalterno frente al trabajo científico general, a la aplicación tecnológica de las ciencias [...] a la fuerza productiva general resultante de la estructuración social de la producción global, fuerza productiva que aparece como don natural del trabajo social (aunque [sea, en realidad, un] producto histórico) ${ }^{6}$ (Marx, 2006, p. 4).

La alteración en la composición del capital determina también las condiciones en las que se produce ciencia, su relación con la producción de tecnología y la forma social como ambas son utilizadas. Marx enfatiza este aspecto: "Las invenciones se convierten entonces en rama de la actividad económica y la aplicación de la ciencia a la producción inmediata misma se torna en un criterio que determina e incita a esta" (Marx 2006, p. 7). Se configura así una ecuación

$6 \quad$ Introduje las cursivas para resaltar la idea. 
inédita según la cual se invierte dinero para obtener conocimiento y se invierte conocimiento para obtener dinero (Perafán 2012, p. 33).

El capital financiero privatizó la tecnología, y en eso radica su poder para producir la globalización económica, productiva, cultural, social y política. Dice Marx:

[...] La acumulación del saber y de la destreza, de las fuerzas productivas generales del cerebro social, es absorbida así, con respecto al trabajo, por el capital y se presenta por ende como propiedad del capital, y más precisamente del capital fixe, en la medida en que éste ingresa como verdadero medio de producción al proceso productivo. La maquinaria, pues, se presenta como la forma más adecuada del capital fixe y el capital fixe -en cuanto se considera al capital en su relación consigo mismo- como la forma más adecuada del capital en general (Marx, 2006, p. 3).

La mano de obra caracterizada por la fuerza física que necesitaba el capitalismo industrial, en el capitalismo actual ha sido superada por la "fuerza-invención" que convierte a la población en una "inteligencia colectiva", en la cual se deposita una "creatividad difusa" que genera una masa de actividad creciente, y que produce trabajo inmaterial. La importancia del capital financiero anida en su capacidad de calcular esos elementos inmateriales que son el corazón del valor de cambio (Moncayo 2010, 57).

Esta dinámica ocupa todo el tiempo de vida y la existencia social (Moncayo 2010, 57, nota al pie N. ${ }^{\circ}$ 3). Marx lo plantea de este modo:

El pilar fundamental de la producción y de la riqueza no es ni el trabajo inmediato ejecutado por el hombre ni el tiempo que éste trabaja, sino la apropiación de su propia fuerza productiva general, su comprensión de la naturaleza y su dominio de la misma gracias a su existencia como cuerpo social; en una palabra, el desarrollo del individuo social [...] Disminuye el tiempo de trabajo en la forma de tiempo de trabajo necesario, para aumentarlo en la forma del trabajo excedente; pone por tanto, en medida creciente, el trabajo excedente como condición [...] del necesario. Por un lado, despierta a la vida todos los poderes de la ciencia y de la naturaleza, así como de la cooperación y del intercambio sociales, para hacer que la creación de la riqueza sea (relativamente) independiente del tiempo de trabajo empleado en ella. Por el otro lado, se propone medir con el tiempo de trabajo esas gigantescas fuerzas sociales creadas de esta suerte y reducirlas a los límites requeridos para que el valor ya creado se conserve como valor. Las fuerzas productivas y las relaciones sociales -unas y otras aspectos diversos del desarrollo del individuo social- se le aparecen al capital únicamente como medios, y no son para él más que medios para producir (Marx 2006, p. 8).

Por lo tanto, la privatización de la tecnología no es la única operación que está en la base de la producción de bienes inmateriales; se recurrió también a la apropiación y sistematización de la cultura que se incorpora y se objetiva en cada sujeto, en forma de conocimiento práctico, de sentido común, de saber hacer, 
es decir, de competencia (o de know-how). El sujeto termina así convirtiéndose en componente de la tecnología.

El desarrollo del capital fixe revela hasta qué punto el conocimiento o knowledge social general se ha convertido en fuerza productiva inmediata, y, por lo tanto, hasta qué punto las condiciones del proceso de la vida social misma han entrado bajo los controles del general intellect y remodeladas conforme al mismo (Marx, 2006, p. 8).

En una economía basada en el conocimiento no basta con desarrollar alta tecnología: hay que aumentar la capacidad de las personas e instituciones en la adquisición, generación, difusión y uso del conocimiento para producir desarrollo social y crecimiento económico (Peluffo y Catalán, 2002, p. 11), pero previniendo

[...] una crisis de sobreproducción, como un exceso de oferta de bienes tecnológicos respecto a la demanda. Un exceso de innovación, una sobreproducción de bienes informacionales (...) La suerte de los bienes informacionales está ligada a la attention economy, a la disponibilidad de un recurso escaso como es la capacidad de atención, de seguimiento de las innovaciones y de socialización de las mismas (Rodríguez y Sánchez, 2004, p. 21) 7 .

\section{Incopporación del conocimiento y el sujeto al proceso de producción}

El capitalismo cognitivo se decanta en el contexto creado por algunas de las tendencias actuales de la expansión y el desarrollo capitalista, al comando del capital financiero. Renán Vega propone a las que siguen como las características del capitalismo cognitivo:

Se consolida una nueva organización de la producción y del trabajo; papel central del conocimiento no sólo como generador de productos intangibles, sino como medio de producción en sí mismo; constitución de una «intelectualidad difusa»; importancia creciente de las tecnologías de información y comunicación (Vega, 2015, pp. 113-116).

El capitalismo cognitivo se concretó por la ocurrencia de cuatro fenómenos muy relacionados entre sí.

El primero es la globalización de un mercado caracterizado por la especialización y la segmentación de la demanda y la presentación e internalización del consumo como factor de la realización y satisfacción personal (Peña, 2014, pp. 99 y ss.). El capitalismo cognitivo es un cambio en la sensibilidad cultural, y afecta la manera de sentir, comprender y conocer. Es una reorganización de la vida humana. Es la manera de significar la vida; por eso el capitalismo cognitivo es exitoso: contaminó el modo mismo del vivir (Ossa, 2012).

7 En este punto puede resultar interesante incluir un dato aportado por Kreimer, citando a Neuman: "Mientras que en 1960 había 98 minutos de información disponible por cada minuto de atención humana, en 2005, cada unidad de atención era disputada por 20.943 minutos de información digital (Neuman et al., 2009)" (Kreimer, 2011, p. 67). 
El segundo fenómeno es el remozamiento funcional de los valores emblemáticos del espíritu liberal clásico, de la modernidad ilustrada, a saber la libertad, el individuo, el derecho, la diferencia ${ }^{8}$. Al neoliberalismo le interesa la legalidad, no la democracia; la legalidad garantiza una libertad negativa entendida por este sistema como individualismo responsable (Grueso, 2007, p. 137) y como libertad para que los individuos consuman y para que el capital pueda invertir sin sobresaltos (Grueso, 2007, p. 138).

La democracia liberal está sustentada en formas universales que tienen como núcleo jurídico y económico la noción de individuo renunciando así a todo contenido colectivo (Chaparro, 2007, 269). En el capitalismo actual han aumentado los derechos formales, pero, a la par, han menguado las condiciones de garantía real de esos derechos y, en consecuencia, ha crecido la intervención del aparato judicial, las relaciones sociales se han juridizado (Ciriza, 2007, p. 305).

El derecho y la política se configuraron como un espacio autonomizado y es allí donde habita el sujeto jurídico que es ahora el ciudadano (Ciriza, 2007, p. 297); quien porta derechos ha sido desmarcado de las singularidades de su origen social, de sus rasgos singulares y de sus determinaciones (Ciriza, 2007, p. 295). Esa abstracción del sujeto jurídico es de gran ayuda en el proceso de entronización del éxito, la felicidad, el hedonismo, la complacencia, el consumo, el vértigo y la satisfacción de los deseos como estados mentales y culturales, ideales o positivos, estados que conducen, de contera, a que más personas crean que está cerca, o que ya se logró, la superación de las necesidades sociales básicas y el aumento del progreso y la democracia.

En las ideas planteadas se manifiestan la ideología liberal clásica y la que es su idealización del individuo; esta concepción del mundo era planteada hace noventa años por Hayek en estos términos:

Los rasgos esenciales del individualismo son: el respeto por el hombre individual qua hombre, es decir, el reconocimiento de sus propias opiniones y gustos como supremos en su propia esfera, por mucho que se estreche esta, y la creencia en que es deseable que los hombres puedan desarrollar sus propias dotes e inclinaciones individuales (Hayek, 2008, 57).

Las virtudes individualistas son

[...] la tolerancia y el respeto para otros individuos y sus opiniones, independencia de juicio y entereza de carácter y disposición para defender sus propias convicciones frente a un superior, la consideración hacia el débil y el enfermo y aquel sano desprecio y desagrado del poder que solo una vieja tradición de libertad personal puede crear [...]

Me refiero a la diferencia promovida como consumo selectivo, como pulsión despolitizada, asociada a exclusividad, envidia, admiración y éxito. Esta forma de diferencia es cuestionada en la categoría "subjetividades blanqueadas" (Piedrahita 2015, 69ss), por sólo mencionar un ejemplo. 
cualidades que facilitan el trato entre hombres en una sociedad libre: cortesía y sentido del humor, modestia personal, respeto a la vida privada de los demás y confianza en las buenas intenciones de su vecino [...] virtudes sociales eminentes, virtudes que suavizan los contactos sociales y que hacen menos necesaria y, a la par, más difícil la intervención desde arriba. Son virtudes que florecen donde ha prevalecido el tipo de sociedad individualista o comercial, y que faltan cuando predomina la sociedad militar o colectivista (Hayek, 2008, 142).

El individualista concluye que debe dejarse a cada individuo, dentro de límites definidos, seguir sus propios valores y preferencias antes que los de otro cualquiera, que el sistema de fines del individuo debe ser supremo dentro de estas esferas y no estar sujeto al dictado de los demás. El reconocimiento del individuo como juez supremo de sus fines, la creencia en que, en lo posible, sus propios fines deben gobernar sus acciones, es lo que constituye la esencia de la posición individualista (Hayek, 2008, p. 86).

La tercera coyuntura fecunda para el capitalismo cognitivo fue el paso de una economía basada en la información a otra basada en el conocimiento y el aprendizaje. La primera tiene su eje central en el manejo, difusión y almacenamiento de la información, las comunicaciones y los datos, y se organiza sobre la base de las tecnologías de la transmisión. La economía del conocimiento, al contrario, se centra en la capacidad de innovar y crear valor con base en el conocimiento y en su rápida actualización en diversos ámbitos por medio del aprendizaje (Peluffo y Catalán, 2002, p. 8). El conocimiento se ha convertido en un factor necesario en el capitalismo moderno, tanto como el trabajo o como el capital,

[...] es un factor intermediario porque "almacena" el valor del trabajo - y de los demás factores productivos- empleado para producirlo. El conocimiento entra en la producción gobernando las máquinas, administrando los procesos y generando utilidad para el consumidor [...] el capital, para valorizarse, debe "subsumir» el conocimiento que genera y que pone en el circuito (Rullani, 2004, p. 100).

En ese marco de "desmaterialización de la mercancía" (Maldonado, 2001, 28) la ganancia obtenida de la valorización del conocimiento se lleva a cabo solo si se puede captar lo cognitivo en favor de lo financiero poniendo cercos al saber; el conocimiento no puede ser de propiedad colectiva sino que debe ser de una empresa para lo cual existen los derechos de propiedad intelectual; lo importante es ampliarlos y prolongarlos en el tiempo para que las empresas puedan amortizar sus costes en I+D (Vercellone, 2004, pp. 69 y 74). Por ello afirma Blondeau: "El refuerzo, por no decir el cerrojazo, de la propiedad intelectual sobre la mercancía es uno de los principales componentes de la estrategia del capital [...] [es la] expresión jurídica de las relaciones de propiedad en el capitalismo informacional" (Blondeau, 2004, p. 37).

En consecuencia, las patentes y los derechos de autor devinieron en instrumentos y estrategias de apropiación de un bien que es colectivo por ser el resultado de un trabajo social largamente realizado. Evans plantea que hay 
[...] un número mayor de productos, que irían de los programas informáticos a las imágenes de los medios electrónicos, [que] son más "ideas» que "cosas». Puesto que el costo de reproducción de una idea es esencialmente cero, los rendimientos aumentan indefinidamente según se expande el ámbito del mercado. En una economía de «ideas», capaz de producir rendimientos crecientes, en vez de "cosas» que producen rendimientos decrecientes, la distribución del ingreso y de los beneficios depende de la posibilidad de su "apropiación». La magnitud de los rendimientos de una idea [...] depende de decisiones discrecionales coercitivas, como la determinación de la duración de los derechos de autor o de la protección de las patentes o del régimen de propiedad intelectual más generalmente (Evans, 2007, p. 113)

Patentes y derechos son los instrumentos que otorgan el poder de competir a las empresas trasnacionales, y de limitar, arrinconar y marginar a los Estados y a los actores sociales de la definición de los rumbos y sentidos de la producción científica y, más importante, de los problemas a los cuales se dedicarán sus científicos e intelectuales. De esa forma se realiza y se fortalece la subordinación de los Estados y sociedades nacionales al capitalismo globalizado y corporativizado ${ }^{9}$ (Vega 2015, 35). En síntesis: "Es suficiente patentar un procedimiento, depositar una idea, inventar un logo, para cobrar los derechos de quien quiera utilizarlos. La creación de riqueza se desplazó de la producción al consumo" (Calderón, 2008, p. 157).

El cuarto fenómeno que dio paso al capitalismo cognitivo es el uso generalizado e intensivo de las NTIC en los procesos de producción de bienes y servicios, en el funcionamiento de las instituciones públicas y privadas, y en la cultura cotidiana. Las NTIC no son hoy simples juguetes tecnológicos para divertimento de las personas adultas, ni herramientas de trabajo; ellas son bienes-saberes cada vez más inutilizables sin la actividad humana viva, que es la única que puede realizar el trabajo de contextualización y que aprovecha su singularidad (Moulier-Boutang, 2004, p. 118).

En esta dinámica la figura del usuario de tecnología se trasmuta en innovador por el uso que hace de los artefactos que utiliza. En efecto:

La utilización es creativa [...] La adopción no es una simple compra. La adopción transforma tanto al que adopta como al objeto adoptado. La adopción es modificadora, puesto que es producción creativa de usos modificadores. El objeto técnico innovador, sea o no NTIC, se transforma y, con frecuencia, de manera crucial, a través de su difusión, de sus adopciones, de sus usos activos. La adopción, la utilización, no es consumo: es producción y, principalmente, producción de usos innovadores (Jollivet, 2004, p. 150).

El internauta y el papel que desempeña también se modifican porque al navegar por la Internet producen una interacción, una forma de relación social,

9 Renán Vega se refiere a estos procesos como "privatización del conocimiento". 
[...] la red es ante todo red humana, y no puede existir sino es a través y por los légamos de la vida que se cristalizan en los artefactos técnicos, a través de la práctica de la multitud de usuarios, productores tanto de sentido como de vida y de los propios artefactos técnicos. Así, uno de los tiempos cruciales de este proceso "legamoso» de innovación por utilización es la incorporación, en el objeto técnico en construcción, de usos innovadores desarrollados a su alrededor por usuarios activos (Jollivet, 2004, p. 151).

\section{Efectos de la entronización del capitalismo cognitivo}

El primero de los varios afectos que se plantean, a continuación, es que la producción contemporánea requiere, del trabajo vivo, capacidades y destrezas para incorporar conocimiento nuevo, trabajar con otras personas y lograr metas; generar la capacidad para entender, controlar, asesorar y mejorar organizaciones y sistemas; resolver conflictos y problemas surgidos en el mundo real; asignar eficientemente los recursos disponibles en relación con los planes y programas estratégicos, administrar tiempo, personas, dinero y recursos materiales y, ahí sí, destrezas técnicas específicas (Peluffo y Catalán, 2002, p. 51).

Ahora es fundamental que la fuerza de trabajo tenga la competencia y la capacidad para utilizar la tecnología y el conocimiento en el desempeño de sus funciones; para incorporarlos en el diseño, la planeación y la ejecución de sus tareas; la disposición interiorizada para mantenerse actualizada y competente en los desarrollos tecnológicos de su campo y, especialmente, que conserve una actitud proclive a la innovación y el cambio. La creatividad es la exigencia que pesa sobre los individuos contemporáneos, creatividad que adquiere formas imprevisibles en un entorno de transformación permanente, de riesgo e incertidumbre. González lo plantea de este modo: "Los profesionales deben conocer lo que se hace para comprender, y anticiparse a las organizaciones con las cuales se compite y para lograr siempre los objetivos en contextos cambiantes; en eso consiste su capital cognitivo" (González, 2008, p. 11).

En este espacio-tiempo predomina un intelecto general que se hace presente en todos los sujetos y que compromete a toda la economía y toda la sociedad. El capitalismo cognitivo se particulariza por la manera como interviene el trabajo vivo en la organización productiva, y porque en esa intervención hacen presencia el conocimiento, la información y las tecnologías asociadas (Moncayo, 2010, p. 61).

Emerge así el intelectual colectivo, el intelecto general, que es un conjunto formado por las "fuerzas productivas", "la cooperación social como acción concertada, por las relaciones "políticas», cognitivas y emotivas, por las capacidades genéricas de hablar, de comprender e imaginar" (Múnera, 2008, 29). Entonces, "la compra de la fuerza de trabajo que constituye el fundamento del capitalismo es la compra de una potencia, de las aptitudes físicas e intelectuales que residen en la corporalidad, y no del simple trabajo efectivo" (Múnera, 2008, 29). El autor puntualiza: 
Se explota directamente el general intellect, inseparable como tal de la vida del trabajador, y por consiguiente, el control de la vida se vuelve más opresivo y represivo, y se realiza no sólo sobre los trabajadores asalariados, sino sobre todas las singularidades productivas, sobre la multitud (Múnera, 2008, p. 30).

En otras palabras "la lógica dominante del capital se rige por los mismos principios de convertir el saber en una fuente de valorización del capital" (Vega, $2015,5)$. En este proceso tiene gran importancia la web porque ella

[...] ha creado un mercado no mercantil del conocimiento y del reconocimiento, así como de la interacción mundializada (...) La Web ofrece un modelo de confrontación entre una oferta y una demanda de conocimientos y de informaciones en tiempo real. La parte no mercantil de este mercado tan particular de la libertad, del juego, del saber, es con mucho la tónica dominante [...] Los portales y los demás motores de búsqueda han sido construidos para recuperar una información y un saber producidos por una multitud de agentes cooperantes sin manufacturas ni empresas, sin capataces ni patronos [...] La cooperación social, determinante en lo que atañe a la producción bajo el régimen del capitalismo cognitivo, incorpora una cantidad considerable de actividad que no es reconocida como trabajo con derecho a remuneración (Moulier-Boutang, 2004, p. 126).

Un segundo efecto es que para ser competitivas y aumentar sus ganancias, las empresas deben incorporar conocimientos, bien sea aumentado la proporción del capital constante representado en desarrollos y aplicaciones tecnológicas de su campo, mejorando la calificación y productividad de su fuerza de trabajo, obteniendo la información necesaria y pertinente para producir innovaciones organizativas, en su producción, su distribución o en la selección/creación de demanda o ampliando su capacidad, habilidad y conectividad para abrirse a nuevos campos y segmentos de mercado. En ese cometido, la universidad adquiere importancia porque es una de las instituciones paradigmáticas; las otras son la empresa y el gobierno, y la relación entre ellas debe ser una concertación interactiva que se acopla a los proyectos, comunicaciones y nuevas clases de valores compartidos (Etzkowiz, 2009, p. 12).

El papel de la universidad en este sistema de innovación, conocido como el modelo de la Triple Hélice, se deriva de ser institución creadora de conocimiento, característica fundamental puesto que el objetivo es el desarrollo económico para lo cual se requiere capital humano en condiciones de adelantar nuevas investigaciones y de gestionar el sistema (Llonto, 2015, p. 2), verdadera "sociedad del conocimiento donde la universidad logra una simbiosis con el tejido empresarial y el Estado" (Llonto, 2015, p. 3). La Triple Hélice ha derivado en un modelo de cuatro hélices; el aspa que se sumó a las tres iniciales fue la sociedad misma en la forma de "organizaciones y personas representativas de la sociedad civil [...] y grupos organizacionales" (Llonto, 2015, p. 3). Este nuevo actor es el sustento estratégico puesto que el propósito final es el crecimiento económico sostenido como condición para el bienestar de la población. 
Varias amenazas pueden entorpecer dicho sistema: la falta de políticas para la educación claras y coherentes, la burocratización de los procesos universitarios que entorpece su acción creadora, la defensa o arraigo de empresas y universidades en objetivos propios que impiden su interacción productiva y eficaz (Llonto, 2015, p. 2). Para conjurarlas es necesario mejorar el capital humano y formar una masa crítica de investigadores que satisfaga las necesidades del sector productivo, y asegurar el desarrollo competitivo de las investigaciones que ayuden a las empresas a alcanzar y elevar las fronteras innovadoras (Llonto, 2015, p. 4). En últimas, el razonamiento es sencillo: la universidad es el factor clave para la investigación y el desarrollo; la empresa aplica el conocimiento y desarrolla un "ecosistema innovador"; el Estado garantiza políticas públicas de fomento a la innovación, la investigación y el desarrollo, y la sociedad sustenta la estrategia (Llonto, 2015, p. 4).

Las políticas públicas se ocupan del desarrollo tecnológico del país, para lo cual reorganizan la acción y gestión institucional más conveniente para sostener las nuevas capacidades de aprendizaje de las empresas, así como de los nuevos agentes que desde diferentes ámbitos (públicos, privados, académicos) contribuyen a desarrollar la diversidad de las capacidades de la sociedad (Casalet, 2006, p. 158). En la economía del conocimiento esta dinámica se manifiesta a través de cuatro aspectos fundamentales: el conocimiento es factor de crecimiento y de progreso; desarrollo de procesos de apropiación social del conocimiento; capacidad de generar procesos de aprendizaje; y mecanismos de Gestión Estratégica del Conocimiento (Peluffo y Catalán, 2002, p. 11). En este contexto es fundamental el apalancamiento del crecimiento económico con políticas públicas de ciencia, tecnología y educación.

Una tercera consecuencia del capitalismo cognitivo es la configuración de entornos competitivos que aumentan las capacidades de las empresas allí localizadas. Estos entornos tienen un carácter regional más que nacional, son producidos por la convergencia en el espacio de empresas competitivas dado el peso de su capital constante, por la capacidad para absorber conocimientos e información y por su inteligencia innovadora. Son agrupaciones de infraestructuras, recursos y organizaciones, auspiciadas por los Estados que permiten la acumulación de colectivos ágiles, flexibles y en ascenso, y generan oportunidades para la innovación. En estos conglomerados se determinan tanto el patrón de competencia al que deben sujetarse empresas menos competitivas, o localizadas en entornos menos potentes, como el lugar y la función que les corresponde realizar en la división del trabajo. Esos entornos son congruentes con las bases de una economía del conocimiento: 
[...] un régimen económico e institucional que procure incentivos al uso eficiente del conocimiento existente, a la generación de nuevos conocimientos y emprendimientos; una población educada y competente que pueda crear y usar el conocimiento de forma efectiva; una infraestructura dinámica de la información y el uso del conocimiento que facilite la comunicación efectiva, la difusión y el procesamiento de la información, y un sistema eficiente de innovación compuesto por una red entre empresas, centros de investigación, universidades, consultoras y otras organizaciones que pueden aumentar el stock de conocimiento global (Peluffo y Catalán, 2002, 11).

Un cuarto corolario hace pie en las concepciones y condiciones de trabajo y de la fuerza laboral. Al respecto plantea Restrepo que en el capitalismo cognitivo la producción se lleva a cabo en condiciones sociales de un alto nivel de desarrollo tecnológico, de sociedades comandadas por máquinas informáticas y sistemas de información masivos, en las que el valor no depende ya de la producción de bienes ni mercancías, sino de la producción de saber y que, por lo tanto, el conocimiento es la genuina fuente de la producción de valor. Se refiere el autor (Restrepo, 2012, Conferencia) al trabajo que realizan los desarrolladores de tecnología o los manipuladores de signos, de la formulación y circulación de consignas, eslóganes y enunciados, de la producción estética, de la producción de discurso, en suma, de un trabajo más abstracto al que llama trabajo cognitivo.

Las condiciones de la producción determinan otros escenarios de trabajo como el laboratorio, otras prácticas como el trabajo ingenieril, y otras formas de vida como las del trabajo deslocalizado o el teletrabajo (Restrepo, 2012, Conferencia). La fuerza laboral sufre un permanente proceso de precarización y explotación que se incrementa a medida que estas formas de trabajo se masifican; se afecta la salud física y mental de la fuerza de trabajo sometida a presión y a un constante estrés psíquico derivado de la explotación de facultades humanas como la atención, el pensamiento y la cooperación (Restrepo 2012, Conferencia).

El capitalismo cognitivo es, en la práctica, una transformación global de los órdenes de explotación y gobierno que tiene que ver con la propiedad intelectual, la guerra global, las cumbres y contracumbres y las grandes manifestaciones (Rodríguez y Sánchez 2004, 28); por lo tanto, está en capacidad de afectar

[...] sectores estratégicos de todas las economías [...] la alimentación y la salud por las patentes sobre la vida y sobre los fármacos; la educación por los procesos de privatización y por la vinculación de la investigación pública a las grandes compañías; el software y la red por las patentes sobre los métodos de programación y por la privatización de internet; los bienes culturales por la aplicación restrictiva y reactiva de los derechos de autor (Rodríguez y Sánchez, 2004, 18).

El capitalismo cognitivo basado en el conocimiento como insumo, medio y producto de la producción; incorporador de "la cultura, la comunicación, la producción lingüística y la producción social de saber cómo medios de producción 
y como productos" (Corsani 2004, 91); animado y concretado por el trabajo incesante del intelecto general en el uso innovador de tecnología; muy competitivo y, a la vez que incluyente, expulsor; ese capitalismo significa, necesariamente, un cambio en las relaciones sociales que está por caracterizarse y frente al cual puede asumirse una postura crítica (quizá catastrofista) o una esperanzada (que puede ser apologética) como la que plantea Blondeau:

Los bienes inmateriales producto del trabajo y de la propiedad común [...] son "mercancías vivas» que se inscriben, a escala planetaria, en el régimen de la propiedad social y del bien común de la humanidad. Expresan, en cuanto tales, una potencia productiva (Blondeau, 2004, 42).

Uno de los elementos esenciales de tal ciclo de producción es "la "presencia del otro". Esta presencia, despojada de toda forma de dependencia y de jerarquía, no es ya una abstracción consubstancial a la mercancía sino la condición misma del acto de producción que se objetiva en ese espacio público de cooperación, que plantea la matriz colectiva como finalidad de la actividad (Blondeau, 2004, 45).

Lejos de disolver el lugar social en un individualismo ciego a la alteridad ¿acaso no inaugura nuevas formas de relación social? [...] En estas condiciones las relaciones sociales, lejos de ser relaciones basadas en la competencia predatoria, se inscriben en una lógica de cooperación reticular ${ }^{10}$. La eficacia económica es entonces el producto de una emulación, de una verdadera movilización colectiva de las inteligencias, que desplaza el centro de gravedad de las relaciones sociales y de sus finalidades (Blondeau, 2004, p. 46).

\section{Conclusiones}

El capitalismo cognitivo es el horizonte en el cual ocurren los procesos educativos, académicos y científicos contemporáneos, al que deben hacer frente las instituciones universitarias, los centros de investigación y desarrollo científico y tecnológico, y las entidades de los Estados encargadas de la educación de sus poblaciones y, por supuesto, de su inserción en los mercados laborales, ahora globalizados. En este marco se agranda la brecha de productividad entre las economías y los actores de importancia mundial y los demás, se define el perfil que debe tener la fuerza de trabajo más calificada, este se hace hegemónico y es reproducido por las políticas para la educación superior, generándose así,

[...] tensiones cada vez más fuertes entre los sistemas educativos de los diferentes países y las demandas, desde el mundo de la vida, por personal calificado, con formaciones pertinentes a la nueva situación y, de otra, tensiones entre los sistemas de investigación y de formación y los nuevos requerimientos del aparato productivo (Misas, 2004, p. 78).

La tensión que expone Gabriel Misas tiene un componente adicional: por un lado, las múltiples necesidades y desafíos que impactan "el mundo de la

10 Esta idea de Blondeau gana factibilidad si se la complementa con otra de Corsani: "[...] son las NTIC las que van a orquestar nuevas formas de cooperación social" (Corsani 2004, 91). 
vida" y que provienen de problemáticas globales como, por ejemplo, el cambio climático, los movimientos migratorios, la seguridad y la soberanía alimentaria, las pandemias generadas por el consumo exacerbado, las tendencias autoritarias y excluyentes que se manifiestan en los sistemas políticos y en la cultura, el desempleo o el empleo precarizado e informal, entre otros, y, por otro lado, la importancia creciente de la actividad económico-productiva determinada por dinámicas cada vez más abstractas y ajenas, por poderes e intereses tan difusos y complejos que se hacen completamente opacos para la percepción y la comprensión social, y hostiles para los Estados.

Esa actividad impone sus necesidades e intereses a las sociedades, los trasmuta en "comunes" y "urgentes" lo cual termina por legitimar que el aparato científico se concentre en darles algún tipo de respuesta, bien sea produciendo conocimiento, solucionando problemas técnico-tecnológicos, o formando capital humano. El involucramiento de la educación superior en este universo económico-productivista fue posible porque la expansión del capital exigía que la educación se relacionara mejor con la productividad y la organización social para la producción, porque el nuevo orden, procedente de las grandes empresas, señala que todo debe estar interrelacionado para participar de un concierto global donde la libre circulación de mercancías requiere estandarizar los requisitos necesarios por las grandes empresas para establecerse en cualquier país (Mejía, 2011, p. 68).

Es en este contexto donde adquiere valor una discusión de importancia estratégica: el sentido de la universidad en la sociedad contemporánea cuando esa institución ha sido escindida entre una responsabilidad hacia sus sociedades nacionales, y la necesidad de ser competitiva en un sistema mundial de universidades del cual es parte cada institución, so pena de ver amenazada su supervivencia misma. En la definición de ese sentido está en juego la sociedad misma en tanto que la complejidad de los problemas políticos, ambientales, sociales y económicos requiere de soluciones y alternativas basadas en procesos intelectuales, pedagógicos e investigativos de los cuales la universidad ha sido el locus histórico. Así las universidades deben tener una doble y complicada orientación: al entorno global y al local, al futuro y al presente.

Entre las nuevas formas de apropiarse de los conocimientos están las que permiten incrementar la competitividad de los individuos y las empresas, inducida por una racionalidad instrumental, por un utilitarismo individualista (Mejía, 2011, 69). A esas formas se unen las apropiaciones del conocimiento que facilita su licenciamiento, que es una forma de expropiarlo a la sociedad, de despojarla de los bienes que producen las instituciones universitarias que 
tienen como fin producirlo para el bien colectivo. En ese contexto, el dispositivo que es el aseguramiento de la calidad, actuó como un instrumento de la globalización dado que fortaleció, e hizo posible, el ideal de los grandes capitales y la consolidación de un mercado, el del conocimiento (Mejía, 2011, 67). Entonces, prácticas ya institucionalizadas en las universidades como la evaluación, la competencia por el financiamiento, la vinculación con el sector productivo, la innovación organizativa y el ordenamiento administrativo son estrategias para modernizar y adecuar las estructuras, los contenidos y la operación de la formación universitaria a los esquemas derivados de la globalización (Mejía, 2011, p. 68).

Este artículo tenía como propósito plantear el capitalismo cognitivo como un entorno importante para pensar las políticas públicas para la educación superior, bajo el supuesto de que los componentes de dicho capitalismo, basado en la incorporación de conocimiento a los procesos productivos, el trabajo inmaterial y la integración de toda la capacidad humana para comunicarse, trabajar de forma colaborativa y así generar conocimiento, afectan a la institución que ha hecho de esa capacidad humana un proceso acumulativo, sistemático y permanente. Asumiendo que se expusieron razones suficientes para demostrar que tal entorno sí existe y es pertinente, queda aún por establecer en qué medida, y cómo, puede determinar al sistema universitario de un país que sigue teniendo una economía basada en la producción de alimentos y minerales, con una tasa de cobertura de la educación superior muy baja, sin presencia en la producción de artículos científicos y patentes, con una desigualdad social que no disminuye, y con una fuerza de trabajo con un nivel medio de escolaridad, bajo salario y más vinculada al sector informal que al formal, entre otras características que lo excluyen de los países industrializados o de Centro en los que el capitalismo cognitivo tiene plena vigencia. En otras palabras, queda por sopesar su alcance explicativo para nuestro caso particular.

\section{Referencias bibliográficas}

Amin, Samir (1999). Capitalismo en la era de la globalización. Buenos Aires: Paidós, Colección Estado y Sociedad.

Asociación Colombiana de Universidades, ASCUN (2007). Políticas y estrategias para la educación superior de Colombia 2006-2010. Bogotá: ASCUN. Recuperado de: http://www.ascun.org.co/publicaciones/detalle/-de-la-exclusion-a-la-equidad-ii-26a

Banco Internacional de Reconstrucción y Fomento/Banco Mundial (2003). La educación terciaria en Colombia. Preparar el terreno para su reforma. Washington: Banco Mundial.

Blondeau, Olivier (2004). "Génesis y subversión del capitalismo informacional". En Olivier Blondeau et al. Capitalismo cognitivo, propiedad intelectual y creación colectiva, pp. 31-48. Madrid: Traficantes de Sueños. 
Boltanski, Luc, y Ève Chiapello (2002). El nuevo espíritu del capitalismo. Madrid: Akal.

Calderón, José (2008). Capitalismo financiero y democracia: Modelos de distribución. Revista Ola Financiera, $\mathrm{N}^{\circ}$. 1, pp. 130-162. Universidad Nacional Autónoma de México, UNAM. Septiembre-diciembre.

Casalet, Mónica (2006). Construcción internacional del mercado en la economía del conocimiento. En Enrique De la Garza. Teorías sociales y estudios del trabajo. Nuevos enfoques, coordinado, pp. 149-158. Barcelona: Anthropos.

Chaparro, Adolfo (2007). La disputa teleológica entre marxismo y liberalismo en los límites de la periferia. En Guillermo Hoyos (Comp.). Filosofía y teorías políticas, entre la crítica y la utopía, por, pp. 257-291. Buenos Aires: CLACSO.

Ciriza, Alejandra (2007). ¿En qué sentido se dice ciudadanía de mujeres? Sobre las paradojas de la abstracción del cuerpo real y el derecho a decidir. En Guillermo Hoyos (Comp.). Filosofía y teorías políticas, entre la crítica y la utopía, pp. 293-319. Buenos Aires: CLACSO.

Corsani, Antonella (2004). "Hacia una renovación de la economía política. Antiguos conceptos e innovación teórica". En Olivier Blondeau et al. Capitalismo cognitivo, propiedad intelectual y creación colectiva, pp. 89-98. Madrid: Traficantes de Sueños.

Di Filippo, Armando (2009). Estructuralismo latinoamericano y teoría económica. Revista CEPAL, N. ${ }^{\circ}$ 98, pp. 198-203.

Dussel, Enrique (2014). 16 tesis de economía política. Interpretación filosófica. México: Siglo XXI Editores.

Etzkowiz, Henry (2009). La triple hélice: universidad, industria y gobierno. Implicaciones para las políticas y la evaluación. Disponible en htpp//:www.sivu.edu.mx/portal/noticias/2009/VinculacionLatriplehelice.pdf. (Consultado 23 de mayo de 2017).

Evans, Peter (2007). Instituciones y desarrollo en la era de la globalización neoliberal. Bogotá: ILSA, Colección En Clave Sur.

González, Pablo (2008). El capitalismo organizado. Entre el orden y el caos. Revista Desacatos, N. ${ }^{\circ} 28$, pp. 165-172. Centro de Investigaciones y Estudios Superiores en Antropología Social. México.

Grueso, Delfín (2007). La globalización y la justicia hacia las identidades culturales. En Guillermo Hoyos (Comp.). Filosofía y teorías políticas, entre la crítica y la utopía, pp. 127-142. Buenos Aires: CLACSO.

Guzmán, César (2008). El disfraz de la competitividad y la producción de conocimientos en el capitalismo cognitivo. Revista Colombiana de Sociología, N. ${ }^{\circ} 31$, pp. 43-60.

Hayek, Friedrich (2008). Camino de la Servidumbre: textos y documentos. Biblioteca de La Libertad. Liberty Fund y ElCato.org. Disponible en htpp://biblioteca.libertyfund.org/sites/default/.../caminode-servidumbre-libro-electronico.pdf. (Consultado 23 de mayo de 2017).

Jollivet, Pascal (2004). Los rendimientos crecientes. En Olivier Blondeau et al. Capitalismo cognitivo, propiedad intelectual y creación colectiva, pp. 149-151. Madrid: Traficantes de Sueños.

Kent, Rollin (2002). Los temas críticos de la educación superior en América Latina en los años noventa. México: Fondo de Cultura Económica.

Kreimer, Pablo (2011). La evaluación de la actividad científica: desde la indagación sociológica a la burocratización. Dilemas actuales. Propuesta Educativa. Vol. 2, N. 36, pp. 59-77. Año 20.

Lazzarato, Maurizio (2004). "Tradición cultural europea y nuevas formas de producción y transmisión del saber". En Olivier Blondeau et al. Capitalismo cognitivo, propiedad intelectual y creación colectiva, pp. 129-144. Madrid: Traficantes de Sueños. 
Llonto, Yefferson (2015). Estado-Universidad-Empresa-Sociedad. Visión de desarrollo económico peruano. Instituto de Economía y Desarrollo -INEDES-Perú. Disponible en https://issuu.com/yeffersonllontocaicedo/docs/el__modelo_de_la_cuadruple_hélice. (Consultado 23 de mayo de 2017).

Maldonado, Carlos (2001). Cotidianidad y destino de la globalización. Bogotá: Universidad Libre, Facultad de Filosofía. Serie Filosofía Política N. 3.

Martínez, Javier (2001). Globalización, elementos para el debate. Una revisión. En Javier Martínez y José Vidal. Crítica de la razón globalizada, pp. 9-44. Quito: Serie Plurimar. ABYA-YALA.

Martínez Pérez, Leonardo Fabio (2012). Ciencia y tecnología en el contexto de la reforma a la Ley de educación superior. En Jornada Académica La medición de grupos de investigación, pp. 28-30. Bogotá: Universidad del Valle, Universidad Distrital, Universidad Pedagógica Nacional.

Marx, Karl. Fragmento sobre las máquinas (2006). Disponible en https://textos.wordpress. com/2006/05/23/fragmento-sobre-las-maquinas. (Consultado 23 de mayo de 2017).

Mejía Montes de Oca, Pablo (2011). Las políticas de aseguramiento de la calidad en la educación superior: una directriz de los organismos internacionales. En Políticas y Educación. La construcción de un destino, coordinadora Sara Rosa Medina, pp. 65-87. México: Posgrados UNAM. CONACYT.

Mignolo, Walter. Sin dato de fecha. Geopolítica del conocimiento y diferencia colonial. Disponible en www.ram-wan.net/.../decolonial/20-mignolo-geopolitica\%20del\%20conocimiento. (Consultado 23 de mayo de 2017).

Misas, Gabriel (2004). La educación superior en Colombia. Análisis y estrategias para su desarrollo. Bogotá: Universidad Nacional de Colombia.

Moncayo, Víctor Manuel (2010). Por una nueva gramática sobre el Estado. Crítica y Emancipación Revista Latinoamericana de Ciencias Sociales. CLACSO, N. . 4, pp. 47 a 79.

Moulier-Boutang, Yann (2004). "Riqueza, propiedad, libertad y renta en el capitalismo cognitivo". En Olivier Blondeau et al. Capitalismo cognitivo, propiedad intelectual y creación colectiva, pp. 107-128. Madrid: Traficantes de Sueños.

Múnera, Leopoldo (2008). "Normalidad y Excepcionalidad en la Política". Disponible en http://www. biopolitica.org/pags/nuestra_red.html. (Consultado 23 de mayo de 2017).

Ordorika, Imanol (2006). Educación superior y globalización: las universidades públicas frente a una nueva hegemonía. Andamios, Vol. 3, N. ${ }^{\circ}$, diciembre, pp. 31-47.

Ordorika, Imanol (2001). Aproximaciones teóricas para un análisis del conflicto y el poder en la educación superior. Perfiles Educativos, Vol. XXIII, N. ${ }^{\circ}$ 91, pp. 77-96. Instituto de Investigaciones sobre la Universidad y la Educación Distrito Federal, México.

Ordorika, Imanol, y Lloyd, Marion (2014). Teorías críticas del Estado y la disputa por la educación superior en la era de la globalización. Perfiles Educativos, Vol. XXXVI, N. ${ }^{\circ}$ 145, pp. 122-139. IISUE-UNAM.

Ossa, Carlos (2012). Conferencia Cultura y Capitalismo Cognitivo (Consejo de la Cultura - Chile) Disponible en https://www.youtube.com/watch?v=Xa4TPFP6-QQ (Consultado 23 de mayo de 2017).

Peluffo, Martha y Edith Catalán (2002). Introducción a la gestión del conocimiento y su aplicación al sector público. Santiago de Chile: Manuales de la CEPAL N. ${ }^{\circ} 22$. Instituto Latinoamericano y del Caribe de Planificación Económica y Social-ILPES. 
Peña Frade, Nayibe (2015). Sistema de aseguramiento de la calidad de la educación superior. Una política pública que merece otra reflexión. Revista Criterio Jurídico Garantista, Vol. 7, N.12, pp. 114 a 145. Universidad Autónoma de Colombia, Facultad de Derecho.

Peña Frade, Nayibe (2014). Intelectuales y universidad: ¿yuxtapuestos, siameses o simbiontes?, Revista Tendencias y Retos, Vol. 19, $\mathrm{N}^{\circ}$. 2, pp. 91-106. Facultad de Ciencias Humanas y Sociales. Universidad de La Salle.

Peña Frade, Nayibe (2013). Colombia 1958-1978: veinte años de políticas para la educación superior. Boletín Observatorio de Desarrollo Humano de la Universidad Autónoma. Primera parte, N. ${ }^{\circ}$ 45, pp. 1-8. Segunda parte, $\mathrm{N}^{\circ} .46$, pp. 1-6. Tercera parte, $\mathrm{N}^{\circ} .47$, pp. 8-11.

Perafán Echeverri, Gerardo Andrés (2012). Dos apuntes con miras a una lectura de los criterios para la medición de grupos de investigación por parte de COLCIENCIAS. En Jornada Académica La medición de grupos de investigación, pp. 31-36. Bogotá: Universidad del Valle, Universidad Distrital, Universidad Pedagógica Nacional.

Piedrahíta, Claudia (2015). Subjetivaciones, subjetividades blanqueadas y nuevas lógicas de la alteridad. En Claudia Piedrahita. Subjetivaciones políticas y pensamiento de la diferencia, pp. 67-78. Bogotá: Biblioteca en Estudios Sociales, Universidad Distrital Francisco José de Caldas.

Restrepo, Carlos Enrique (2012). La reapropiación social del conocimiento: tareas de la universidad por venir. Conferencia en Universidad de Antioquia. Disponible en https://filoficciones.wordpress. com/2012/06/12/la-reapropiacion-social-del-conocimiento-tareas-de-la-universidad-porvenir/ (Consultado 23 de mayo de 2017).

Rodríguez, Emmanuel y Sánchez, Raúl (2004). Entre el capitalismo cognitivo y el Commonfare. En Olivier Blondeau et al. Capitalismo cognitivo, propiedad intelectual y creación colectiva, pp. 13-28. Madrid: Traficantes de Sueños.

Rullani, Enzo (2004). "El capitalismo cognitivo ¿un déjà-vu?" En Olivier Blondeau et al. Capitalismo cognitivo, propiedad intelectual y creación colectiva, pp. 99-106. Madrid: Traficantes de Sueños.

UNESCO-CRESALC, ASCUN (1998). Políticas y estrategias para la transformación de la educación superior en América Latina y el Caribe. Hacia una agenda de la educación superior en Colombia. Bogotá: Memorias.

Vega, Renán (2015). La universidad de la ignorancia. Capitalismo académico y mercantilización de la educación superior. Bogotá: Océano Sur.

Vercellone, Carlo (2004). "Las políticas de desarrollo en tiempos del capitalismo cognitivo". En Olivier Blondeau, et al. Capitalismo cognitivo, propiedad intelectual y creación colectiva, pp. 63-74. Madrid: Traficantes de Sueños. 